\title{
DESIGN AND IMPLEMENTATION OF MULTIPLIER-LesS TUNABLE 2-D FIR FILTERS USING MCCLELLAN TRANSFORMATION
}

\author{
K. S. Yeung and S. C. Chan
}

Department of Electrical and Electronic Engineering, The University of Hong Kong, Pokfulam Road, Hong Kong.

\begin{abstract}
This paper proposes new structures for realizing tunable 2-D fan and elliptical filters with different spectral characteristics using McClellan transformation. The 1-D prototype is a variable digital filter obtained from the interpolation of a set of desirable impulse responses and is implemented using the Farrow structure. The coefficients of the sub-filters and the transformation parameters in the Farrow structure are represented in SOPOT, which can be easily implemented as simple shift-and-add operations. Furthermore, the transformation part can be shared between the sub-filters leading to significant saving in hardware complexity. Several design examples are given to demonstrate the effectiveness and feasibility of the proposed approach.
\end{abstract}

\section{INTRODUCTION}

Two dimensions (2-D) digital filters with variable or tunable spectral characteristics are useful in video signal processing, array signal processing [9], image processing and analysis [10], and computer vision [11]. Methods for designing one-dimensional (1D) variable digital filters (VDF) are studied in [12] and it can broadly be classified into two categories: transformation [13] and spectral parameter approximation [14] methods. In the former, a prototype filter with certain desirable spectral characteristics is first designed. Certain transformation such as the allpass transformation method is then applied to the prototype filter to obtain the final VDF. The spectral parameter approximation method is more general in that it assumes that either the impulse responses or the poles and zeros of the filters are polynomials of certain spectral parameters. The coefficients of the polynomials are then determined to provide continuous tuning of the VDF by the spectral parameters. Although the spectral parameter method can be generalized to two or higher dimensions, its complexity is rather high. For certain 2-D digital filters such as the circular symmetric filters with variable cutoff frequencies, fan filters with variable inclination and filter support, and elliptical filters with different orientation and filter support, it is advantageous to transform the 1$D$ variable digital structure to higher dimensions using the McClellan transformation [1]-[5], greatly reducing the design and implementation complexity of the 2-D VDF. The design and implementation of such 2-D circular symmetric filters with variable cutoff frequencies were studied recently by Pun et al [6].

In this paper, the work in [6] is further extended to the design and implementation of variable 2-D fan and elliptical filters. First of all, a 1-D VDF with the desired tunable range of cutoff frequencies is designed and is implemented using the Farrow structure [6]. Then, the sub-filters in the Farrow structure will undergo the McClellan transformation to obtain the variable 2-D fan or elliptical filters. By varying simultaneously the control parameter of the Farrow structure (which determines the cutoff frequencies of the prototype) and the transformation parameters (which determines the orientation, etc), the inclination/orientation and support of these fan or elliptical filters can be varied on-line. Because the coefficients in the sub-filters are fixed, they can be efficiently implemented using sum-of-powers-of-two (SOPOT) coefficients or canonical signed digits (CSD) yielding multiplierless realization. Since the McClellan transformation is applied to all the sub-filters, the transformation part can be shared among different sub-filters, representing significant saving in hardware complexity. For a given inclination/orientation, the transformation parameters are first generated by linear interpolation and are rounded to appropriate SOPOT coefficients. All the building blocks for implementing the McClellan transformation are then configured to implement these SOPOT transform parameters. Since the transform parameters are very few, such building blocks are relatively easy to implement. The final VDF structure requires very few general-purpose multipliers, which is equal to the order of interpolation used and it can be keep to a small number by dividing the tuning range into smaller non-overlapping sections, if necessary.

The rest of this paper is organized as follows: Section II is devoted to the theory and design of 2-D zero-phase FIR filters using McClellan transformation. Section IIl presents the design of tunable 2-D FIR filters using the Farrow structure. Section IV describes their multiplier-less realization for the sub-filters, followed by several design examples in Section V. Finally, conclusion is drawn in Section VI.

\section{2-D FIR FILTER DESIGN USING MCCLELLAN TRANSFORMATION}

Consider a zero-phase FIR digital filter [8] with impulse response $h(n)=h(-n),-N \leq n \leq N$. Then we can write

$$
H(\omega)=\sum_{n=0}^{N} b(n) \cos (\omega n) .
$$

where $b(n)$ is equals to $h(0)$ when $n=0$, and $2 h(0)$ when $n \neq 0$. By expressing the function $\cos (\omega n)$ as a polynomial of degree $n$ in the variable $\cos (\omega), H(\omega)$ can be rewritten as

$$
H(\omega)=\sum_{n=0}^{N} b(n) T_{n}[\cos (\omega)]
$$

where $T_{n}[\cdot]$ is the $n^{\text {th }}$ order Chebyshev polynomial. The idea of McClellan transformation is to replace the function $\cos (\omega)$ by a 2 D function of $\left(\omega_{1}, \omega_{2}\right)$, denoted as $F\left(\omega_{1}, \omega_{2}\right)$, so that the 1-D frequency response of $H(\omega)$ will be mapped to the $\left(\omega_{1}, \omega_{2}\right)$-plane according to the contour specified by $F\left(\omega_{1}, \omega_{2}\right)$. That is

$$
H\left(\omega_{1}, \omega_{2}\right)=\sum_{n=0}^{N} b(n) T_{n}\left[F\left(\omega_{1}, \omega_{2}\right)\right] .
$$

\section{A. 2-D Fan Filters}

To design a 2-D fan filter with arbitrary inclination, the following first-order McClellan transformation with $\left|F\left(\omega_{1}, \omega_{2}\right)\right| \leq 1$ can be used

$$
\begin{aligned}
\cos (\omega)= & t(0,0)+t(1,0) \cos \left(\omega_{1}\right) \\
& +t(0,1) \cos \left(\omega_{2}\right)+t(1,1) \cos \left(\omega_{1}\right) \cos \left(\omega_{2}\right)
\end{aligned}
$$

According to [4], it is desired for the transformation to satisfy the following constraints: 1) the 1-D frequency origin, $\omega=0$, maps to the $(0, \pi)$-point of the 2-D frequency plane, and 2) the point $\omega=\pi$ in 1-D maps to the $(\pi, 0)$-point of the 2-D frequency plane to avoid the scaling-problem of the transformation and to reduce 
the number of free parameters. It can be shown that these two constraints are satisfied if $t(0,0)=t(1,1)$ and $t(1,0)=1+t(0,1)$. Hence, (7) becomes

$$
\begin{aligned}
\cos (\omega)= & t(1,1)\left(1+\cos \left(\omega_{1}\right) \cos \left(\omega_{2}\right)\right) \\
& +t(0,1)\left(\cos \left(\omega_{1}\right)+\cos \left(\omega_{2}\right)\right)+\cos \left(\omega_{1}\right)
\end{aligned}
$$

Assume $\omega_{c}$ is the cutoff frequency of the 1-D prototype filter and $\theta$ is the inclination angle of the ideal 2-D fan filter. Thus, (5) should map the 1-D cutoff frequency at $\omega_{c}$ to the line $\omega_{2}=\omega_{1} \tan (\theta)$ [5]. The transform parameters can be determined by minimizing the following mean-squares error (MSE) function

$$
e=\int_{b p}^{\omega_{u p}}\left|\cos \left(\omega_{\mathrm{c}}\right)-F\left(\omega_{1}, \omega_{2}\right)\right|^{2} d \omega_{1},
$$

where $\omega_{u p}=\left\{\begin{array}{lc}\pi, & 0^{\circ}<\theta<45^{\circ} \\ \pi / \tan (\theta), & 45^{\circ}<\theta<90^{\circ}\end{array}\right.$. The MSE function can be rewritten in a Quadratic form as

$$
e=s+P^{\prime} T+T^{\prime} P+T^{\prime} Q T,
$$

where $\boldsymbol{P}=\int_{0}^{\omega_{\varphi}}-\left(\cos \left(\omega_{c}\right)-\cos \left(\omega_{1}\right)\right) \cdot C d \omega_{1}$,

$\boldsymbol{s}=\int_{0}^{\omega_{\oplus}}\left(\cos \left(\omega_{c}\right)-\cos \left(\omega_{1}\right)\right)^{2} d \omega_{1}, Q=\int_{0}^{\omega_{\infty}} C C^{\prime} d \omega_{1}, T=[t(1,1), t(0,1)]^{t}$,

$\boldsymbol{C}=\left[1+\cos \left(\omega_{1}\right) \cos \left(\omega_{2}\right), \cos \left(\omega_{1}\right)+\cos \left(\omega_{2}\right)\right]^{t}$,

which has the minimum value at

$$
T=-Q^{-1} P
$$

Using (8), we can compute the corresponding transformation parameters and cutoff frequencies for a given inclination of the fan filter. These parameters can be used to control the tuning parameters in the variable 2-D fan filter to be described in Section III to provide on-line tuning.

\section{B. 2-D Elliptical Filters with Arbitrary Orientation}

To design 2-D elliptical filters with arbitrary orientation $\theta$, the following McClellan transformation

$$
\begin{aligned}
\cos (\omega)= & t(0,0)+t(1,0) \cos \left(\omega_{1}\right)+t(0,1) \cos \left(\omega_{2}\right) \\
& +t(1,1) \cos \left(\omega_{1}\right) \cos \left(\omega_{2}\right)+s(1,1) \sin \left(\omega_{1}\right) \sin \left(\omega_{2}\right)
\end{aligned}
$$

is employed [4]. The cutoff frequency boundary of the rotated ellipse can be described as

$$
\frac{\omega_{1}^{2}}{A^{2}}+\frac{\omega_{2}^{2}}{B^{2}}+\frac{\omega_{1} \omega_{2}}{C}=1,
$$

where $A^{2}=a^{2} / D_{0}, B^{2}=b^{2} / D_{0}, C=a b / D_{1}$;

$$
D_{0}=\left[1-\left(1-(a / b)^{2}\right) \sin ^{2}(\theta)\right]^{-1}, D_{1}=[(b / a)-(a / b)] \sin (2 \theta) .
$$

The constants $a$ and $b$ are the cutoff frequencies on the $\omega_{1}$ - and $\omega_{2}$-axis with $\theta=0$. For $b>a$, two constraints have to be satisfied [4]: 1) $\omega=0$, should map to the origin $(0,0)$, and 2) $\omega=\pi$ should map to the point $(\pi, 0)$. This gives $t(0,1)=-t(0,0)$, and $t(1,1)=1$ $t(1,0)$. Hence, $(9)$ becomes

$$
\begin{aligned}
\cos (\omega)= & \cos \left(\omega_{1}\right) \cos \left(\omega_{2}\right)+t(0,0)\left(1-\cos \left(\omega_{2}\right)\right) \\
& +t(1,0)\left(\cos \left(\omega_{1}\right)-\cos \left(\omega_{1}\right) \cos \left(\omega_{2}\right)\right) . \\
& +s(1,1) \sin \left(\omega_{1}\right) \sin \left(\omega_{2}\right)
\end{aligned}
$$

The technique described above [3] is used to find the optimum transformation coefficients and the corresponding cutoff frequency $\omega_{c}$ of the 1-D prototype filter. The variables of the MSE function (7) in this design are

$$
\begin{aligned}
& s=\int_{A}^{1}\left(\cos \left(\omega_{c}\right)-\cos \left(\omega_{1}\right) \cos \left(\omega_{2}\right)\right)^{2} d \omega_{1} \\
& P=\int_{A}^{A}-\left(\cos \left(\omega_{c}\right)-\cos \left(\omega_{1}\right) \cos \left(\omega_{2}\right)\right) \cdot \boldsymbol{C} \cdot d \omega_{1} \\
& \boldsymbol{Q}=\int_{A}^{1} C C^{t} d \omega_{1}, \boldsymbol{T}=[t(0,0), t(1,0), s(1,1)]^{t} \\
& \boldsymbol{C}=\left[1-\cos \left(\omega_{2}\right), \cos \left(\omega_{1}\right)-\cos \left(\omega_{1}\right) \cos \left(\omega_{2}\right), \sin \left(\omega_{1}\right) \sin \left(\omega_{2}\right)\right]^{\prime}
\end{aligned}
$$

Again, we can compute the corresponding transformation parameters and cutoff frequencies for different orientations of the elliptical filter. These parameters can be used to control the tuning parameters for the variable 2-D elliptical filter to provide on-line tuning.

\section{DESIGN OF 1-D PROTOTYPE FILTER WITH VARIABLE CUTOFF FREQUENCIES}

We now describe the design of the 1-D prototype filter with variable cutoff frequencies. Assume that the impulse response of the filter with cutoff frequency $\omega_{c}$ is associated with some control parameter $\phi$ and is given by

$$
H(z, \phi)=\sum_{n=0}^{2 N} h(n, \phi) z^{-n}
$$

$h(n, \phi)$ is assumed to be an $L^{\text {th }}$ order polynomial in variable $\phi$

$$
h(n, \phi)=\sum_{l=0}^{L} c_{l, n} \phi^{l}
$$

This technique was first proposed by Farrow [7] for the design of fractional-delay digital filters. The $z$-transform of (14) is

$$
H(z, \phi)=\sum_{l=0}^{L}\left[\sum_{n=0}^{2 N} c_{l, n} z^{-n}\right] \phi^{l}=\sum_{l=0}^{L} C_{l}(z) \phi^{\prime}
$$

where $C_{l}(z)=\sum_{n=0}^{2 N} c_{l, n} z^{-n}$ are called the sub-filters. The coefficients $c_{l, n}$ are determined by polynomial interpolating the impulse response $h(n, \phi)$ designed at regular interval of $\phi$. Alternatively, it can be obtained in using a least squares approach [12]. By transforming $C_{l}(z)$ as in (3), tunable 2-D zero-phase FIR filter with control or tuning parameter $\phi$ can be implemented by a similar structure as shown in Fig. 1. The transformed sub-filters are

$$
C_{l}\left(z_{1}, z_{2}\right)=\sum_{n=0}^{N} b_{l}(n) T_{n}\left[F\left(z_{1}, z_{2}\right)\right],
$$

while the tunable 2-D FIR filter is

$$
H\left(z_{1}, z_{2}, \phi\right)=\sum_{l=0}^{L} C_{l}\left(z_{1}, z_{2}\right) \phi^{\prime} .
$$

It can be implemented by the structure shown in Fig. 1. Note that the same McClellan transformation $F\left(z_{1}, z_{2}\right)$ is applied to all the sub-filters. Therefore, it can be shared among the sub-filters representing significant hardware saving, especially in our case where the transform parameters are also varied according to the tuning parameters, unlike the circular case in [6]. The transformation coefficients are realized using linear approximation as a function of tuning parameter $\phi$, i.e. the first order of interpolation. Its output is then quantized to SOPOT coefficients to configure the transformation block $F$ in Fig. 1.

\section{MULTIPLIER-LESS SUB-FILTERS}

To reduce the implementation complexity of the 2-D VDF, the coefficients of the sub-filters and the transformation parameters in the transformation block $F$ are implemented as SOPOT coefficients in the form

$$
\hat{b}_{l}(n)=\sum_{r=1}^{R} u_{l, n, r} 2^{a_{r}}
$$


with $u_{l, n, r} \in\{-1,1\}$ and $a_{r} \in\{-g, \ldots,-1,0,1, \ldots, g\}$, where $g$ is a positive integer and its value determines the range of the coefficients, and $R$ is the number of terms used in the coefficient approximation and is usually limited to a small number. The coefficient multiplication can then be implemented as limited number of shifts and additions. The generation of the SOPOT transformation parameters has been described in previous section. To determine the SOPOT coefficient for the sub-filters, we minimize the peak ripple error $\delta$

$$
\delta=\max \left(\left|H_{i}(\omega)-\hat{H}(\omega)\right|\right),
$$

between the ideal frequency response $H_{i}(\omega)$ and the frequency response $\hat{H}(\omega)$ of the given SOPOT coefficients. In other words, we try to minimize the peak ripple error $\delta$ for the whole frequency range of interest and the whole tunable range $\left(\omega_{c 2}-\omega_{c 1}\right)$. The real-valued coefficients $b_{l}(n)$ are first determined as mentioned earlier. Let $b$ be the vector containing these all coefficients. The random search algorithm is to repetitively calculate a candidate SOPOT vector $\boldsymbol{b}_{c}$ defined by

$$
\boldsymbol{b}_{c}=\left|\boldsymbol{b}+\lambda \boldsymbol{b}_{p}\right|_{\text {SOPOT }}
$$

where $\boldsymbol{b}_{p}$ is a random vector with elements chosen in the range \pm 1 , $\lambda$ is a user-defined variable used to control the size of the neighborhood to be searched, and []$_{\text {SOPOT }}$ is the rounding operator that converts every element inside the input vector to its closest SOPOT value with a given value of $g$. The performance measures $\delta$ of the new coefficients are then calculated. The set that yields the minimum peak ripple error $\delta$ under the given constraints of total number of terms and $g$ is the optimum solution. Since this is a random search algorithm, the longer the searching time, the higher the chance of finding the optimal solution. There are two advantages of this algorithm. First of all, with the computational power of nowadays personal computer (PC), the time for obtaining high quality solution is manageable. The other one is applicable to problem with general objective function probably with very complicated inequality constraints. Moreover, a set of possible solutions representing different tradeoffs between computational complexity and performance will be generated during the search. We now present several design examples.

\section{Design EXAMples}

\section{Example 1. 2-D Fan Filter with Tunable Inclination}

In this example, a 2-D zero-phase FIR fan filter with tunable inclination from $25^{\circ}$ to $35^{\circ}$ is designed. The corresponding cutoff frequency of 1-D prototype filter is from $0.75 \pi$ down to $0.64 \pi$. The transition bandwidth is $0.1 \pi$. The order of the sub-filters is 30 , i.e. $N=15$ and the order of the interpolation polynomial $L$ is 2 . The worst-case stopband attenuation is around $30 \mathrm{~dB}$. The realvalued coefficients of sub-filters and that of the transformation require 48 and 60 multipliers, respectively. The multiplier-less (SOPOT) sub-filters and transformation, however, require respectively 49 and 90 adders instead. For larger tuning range, say from $25^{\circ}$ to $45^{\circ}$, we can divide the tuning range into two parts, one from $25^{\circ}$ to $35^{\circ}$ and the other from $35^{\circ}$ to $45^{\circ}$. A similar set of subfilters can be designed and will operate only within the new tuning range from $35^{\circ}$ to $45^{\circ}$. The contour plots of the frequency responses of the tunable 2-D fan filter with variable inclination are shown in Fig. 2. Since the transformation part and the interpolation part (with $\phi$ ) in Fig. 1 are both common to the two sections, only the constant multiplications $\hat{b}_{l}(n)$ need to be implemented for the new section. Furthermore, the corresponding constant multiplications for different sections can be implemented together using the technique of multiplier block.

\section{Example 2. 2-D Elliptical Filter with Tunable Orientation}

In this example, a 2-D zero-phase FIR elliptical filter with tunable orientation from $15^{\circ}$ to $30^{\circ}$ is designed with fixed $a=$ $0.125 \pi$ and $b=0.375 \pi$. The corresponding cutoff frequency of 1-D prototype filter is from $0.129 \pi$ to $0.143 \pi$. The transition bandwidth is $0.1 \pi$. The order of the sub-filters is 30 , i.e. $N=15$ and the order of the interpolation polynomial $L$ is 2 . The worstcase stopband attenuation is around $30 \mathrm{~dB}$. The real-valued coefficients of sub-filters and that of the transformation require 48 and 90 multipliers, respectively. The multiplier-less realization of the sub-filters and that of the transformation require only 38 and 90 adders, respectively. The contour plots of the frequency responses of the tunable 2-D elliptical filter with orientation at $15^{\circ}, 22.5^{\circ}$, and $30^{\circ}(\phi=0,0.5,1)$ are shown in Fig. 3. Alternatively, the elliptical size of this filter can be varied with fixed orientation.

\section{CONCLUSION}

New structures for realizing tunable 2-D fan and elliptical filters with different spectral characteristics using McClellan transformation are presented. The 1-D prototype is a VDF obtained from the interpolation of a set of desirable impulse responses and is implemented using the Farrow structure. The coefficients of the sub-filters and the transformation parameters in the Farrow structure are represented in SOPOT, which can be easily implemented as simple shift-and-add operations. Furthermore, the transformation part can be shared between the sub-filters leading to significant saving in hardware complexity. Several design examples have given to demonstrate the effectiveness and feasibility of the proposed approach.

\section{REFERENCES}

[1] J. H. McClellan, "The design of two-dimensional digital filters by transformation," in Proc. $7^{\text {th }}$ Annual Princetion Conference. Information Sciences and Systems 1973. pp. 247-251.

[2] Y. S. Song and Y. H. Lee, "Formulas for McClellan transform parameters in designing 2-D zero-phase FIR Fan Filters," IEEE Signal Processing Lett., vol. 3, no. 11, pp. 291 293, Nov 1996

[3] H. C. Lu and K. H. Yeh, "2-D FIR filters design using least square error with scaling-free McClellan transformation," IEEE Trans. Circuits Syst. II, vol. 47, no. 10, Oct 2000, pp. 1104-1107.

[4] D. T. Nguyen and M. N. S. Swamy, "Formulas for parameters scaling in the McClellan transform," IEEE Trans. Circuits Syst,, vol. CAS-33, pp. 108-109, 1986.

[5] E. Z. Psarkis V. G. Mertzios, and F.P.H. Alexiou, "Design of twodimensional zero phase FIR fan filtes via the McClellan transform," IEEE Trans. Circuits Syst., vol. 37, pp. 10-16, 1990.

[6] C. K. S. Pun S. C. Chan, and K.L. Ho, "Efficient 1D and circular symmetric 2D FIR filters with variable cutoff frequencies using the farrow structure and multiplier-block," in Proc. IEEE ISCAS'2001, vol. 2, pp. 561-564.

[7] C. W. Farrow, "A continuously variable digital delay element," in Proc. IEEE ISCAS'1988, pp. 2641-2645

[8] D. E. Dudgeon and R. M. Mersereau, Multidimensional digital signal processing, Englewood Cliffs, NJ: Prentice-Hall, 1984.

[9] A. C. Tan et al, S. T. Chen and S. Basu, "Adaptive wave digital FAN filters," IEEE ICASSP-1994, vol. 6, pp. 25-28.

[10] T. L. Marzetta, "The role of the velocity-slowness mapping in FAN filtering of image sequences," IEEE ICASSP-1992, vol. $3 \mathrm{pp}$. 433-435

[11] W. T. Freeman and E. A. Adelson, "The design and use of steerable filters," IEEE Trans. on Pattern Anal. Machine Intell., vol. 13, no. 9, Sept. 1991, pp. 891-906.

[12] S. C. Chan, K. S. Pun and K. L. Ho, "On the design and implementation of FIR and IIR digital filters with variable frequency characteristics," Internal report, The University of Hong Kong, Sept. 2001.

[13] P. Jarske, Y. Neuvo; and S. K. Mitra, "A simple approach to the design of linear phase FIR digital filters with variable characteristics," IEEE Trans. Signal Processing, vol. 14, no. 4, pp. 313-326, June 1988.

[14] T. B. Deng, "Design of recursive 1-D variable filters with guaranteed stability," IEEE Trans. Circuits Syst. II, vol. 9, pp. 689-695, Sept 1997. 
Transformation part

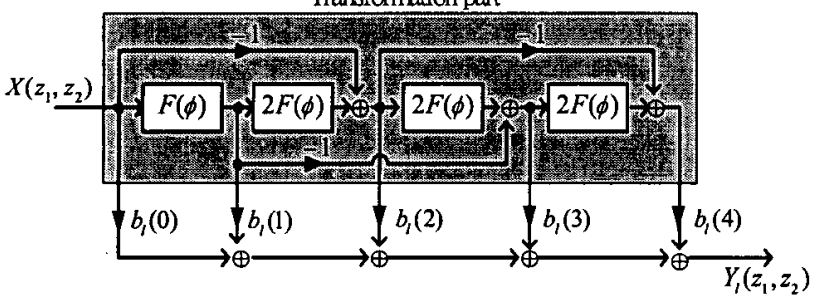

(a)

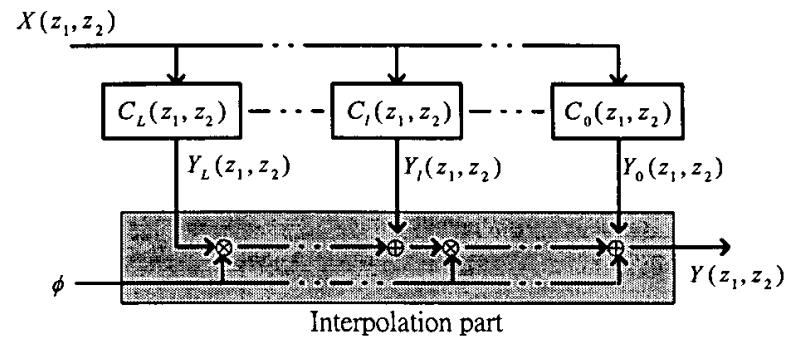

(b)

Fig. 1. Proposed structure of tunable 2-D FIR filters (a) Sub-filter $C_{l}\left(z_{1}, z_{2}\right)$ realization (b) Proposed Farrow structure

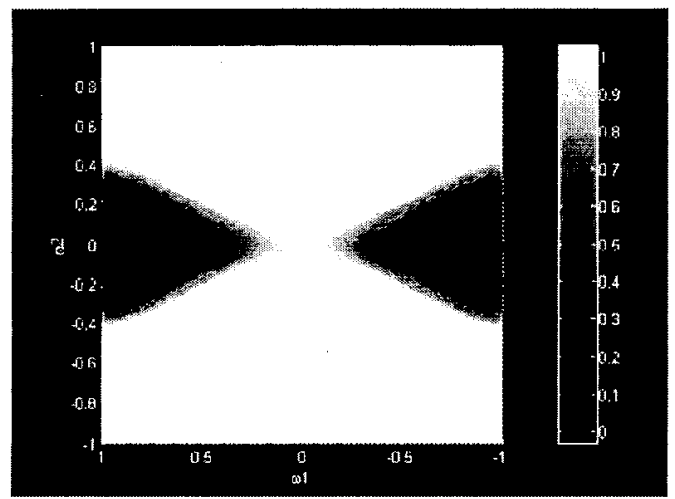

(a)

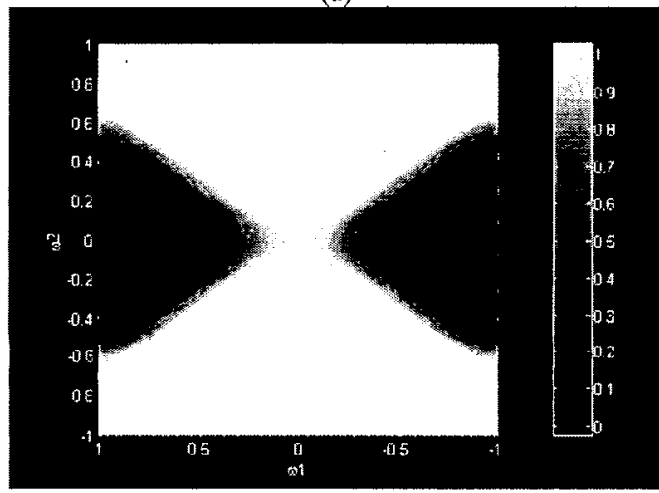

(b)

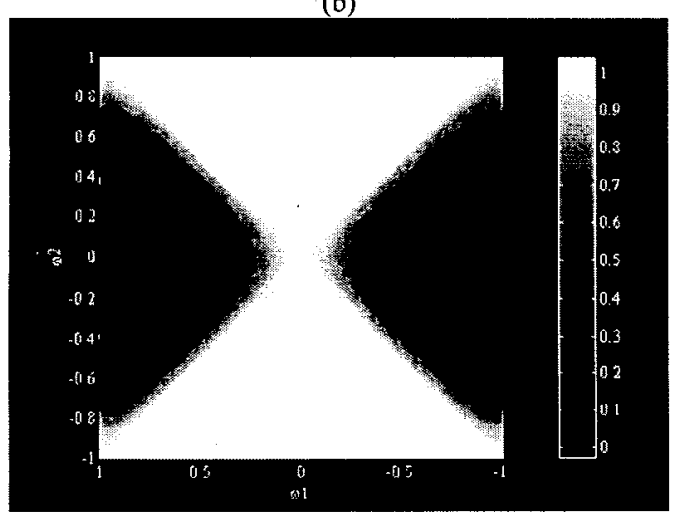

(c)

Fig. 2. Contour plots of frequency responses of tunable 2-D fan filter (divided into two sections) with inclination at (a) $25^{\circ}$, (b) $35^{\circ}$, and (c) $45^{\circ}$.

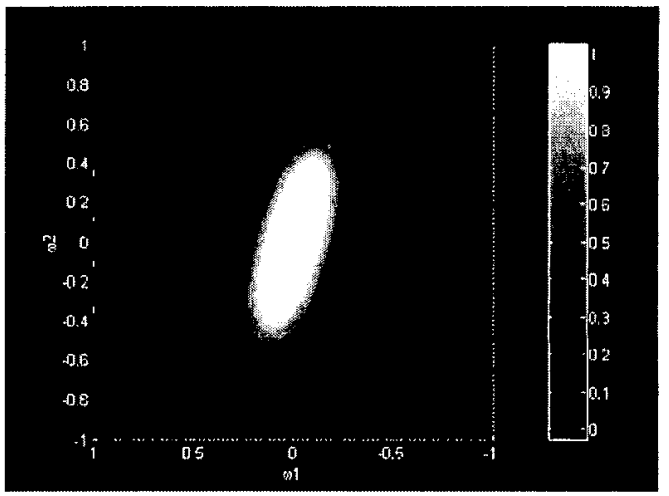

(a)

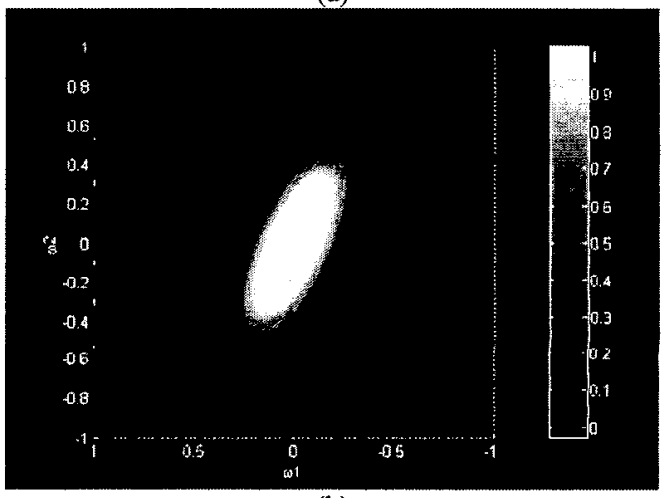

(b)

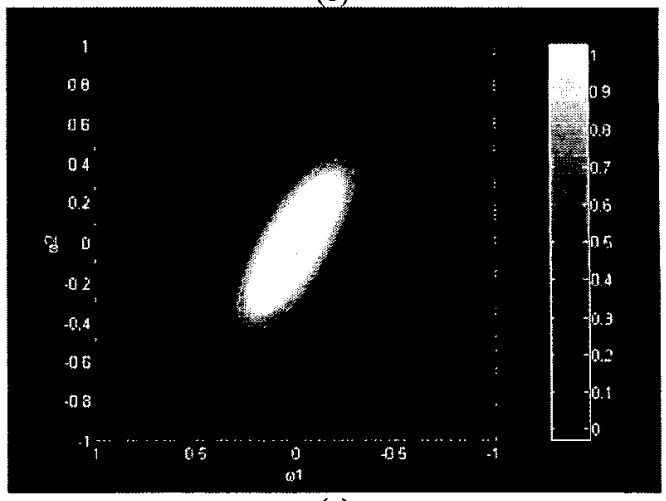

(c)

Fig. 3. Contour plots of frequency responses of tunable 2-D elliptical filter with orientation at (a) $15^{\circ}$, (b) $22.5^{\circ}$, and (c) $30^{\circ}(\phi=0,0.5,1)$. 